\title{
Impact of high-fat diet on antioxidant status, vascular wall thickening and cardiac function in adult female $\mathbf{L D L R}^{-/}$ mice
}

\author{
Stéphanie Delemasure ${ }^{1,2}$, Carole Richard ${ }^{2,3}$, Ségolène Gambert ${ }^{4}$, Jean-Claude Guilland ${ }^{2,4}$, \\ Catherine Vergely ${ }^{2}$, Patrick Dutartre ${ }^{1,2}$, Luc Rochette ${ }^{1,2}$, Jean-Louis Connat ${ }^{1,2^{*}}$ \\ ${ }^{1}$ COHIRO Biotechnology, Faculty of Medicine, Dijon, France \\ ${ }^{2}$ Laboratory of Cardio-Metabolic Pathophysiology and Pharmacology, Faculty of Medicine, University of Burgundy, Inserm UMR866, \\ Dijon, France \\ ${ }^{3}$ Department of Cardiology, University Hospital, Dijon, France \\ ${ }^{4}$ Biochemistry Department, University Hospital, Dijon, France \\ Email: *connatjl@u-bourgogne.fr
}

Received 24 April 2012; revised 24 May 2012; accepted 29 May 2012

\begin{abstract}
Background: Western diet, rich in saturated fatty acids and cholesterol, is associated with increased cardiovascular risk. We thus investigated in female mice the influence of this diet on plasma antioxidant status, vascular wall thickening and cardiac function. Methods and Results: Adult female C57BL/6J wild type (WT) and $\mathrm{LDLR}^{-/-}$mice were fed a normal diet (ND) or a high-fat diet (HFD) for 17 weeks. HFD induced an increase in plasma lipids and vitamin C (Vit C) levels in both groups but at a much higher level in $\mathbf{L D L R}^{-/-}$and a decrease in plasma ascorbyl free radical levels to Vit $C$ ratio (an endogenous oxidative stress index) in $\mathbf{L D L R}^{-/-}$. We only found a slight decrease in circulating antioxidant status evaluated by the Oxygen Radical Absorbance Capacity (ORAC) assay in WT, but not in LDLR $^{-/-}$. Echocardiography evidenced an increase in arterial wall thickness in aortic arch at atherosclerosis predilection sites in ${\text { HFD } \text { LDLR }^{-/-} \text {as compared to ND LDLR }}^{-/}$and HFD WT. This result was confirmed by histology. Furthermore, histological examination of aortic valves showed an increase in atherosclerotic lesions. Our study, using echocardiography, show that chronic HFD does not induce any major modifications of systolic function in the both mice groups. Conclusions: High-fat intake in mice causes serious disturbances in lipid plasma levels associated to variations of circulating antioxidant status due, at least in part, to an increase in Vit C. At this stage, atherosclerotic lesions, observed in aortic arch and valve, do not impair cardiac function in HFD-fed mice.
\end{abstract}

\footnotetext{
"Corresponding author.
}

Keywords: Ascorbyl Free Radical; Echocardiography; Vitamin C; High-Fat Diet; Oxygen Radical Absorbance Capacity; Oxidant/Antioxidant Status; Systolic Function; Aortic Arch

\section{INTRODUCTION}

Western type high-fat diet (HFD), typically rich in saturated fatty acids and cholesterol, is associated with increased cardiovascular risk due, in part, to an abnormal increase in plasma cholesterol [1]. Low density lipoproteins (LDL) are known to promote the initiation of atherosclerotic vascular disease. Lipoprotein metabolism may be disturbed experimentally by using geneticallymodified animal models. The inactivation of the LDL receptor $\left(\mathrm{LDLR}^{-1}\right)$ in mice allowed to reproduce hypercholesterolemia and massive increase of circulating LDL [2]. LDLR ${ }^{-/}$mice develop lipid lesions, similar to those found in humans, mainly localized at the aortic arch, when fed under atherogenic diet but not under normal low-fat diet (ND) [3].

Atherogenesis is a chronic inflammatory process that involves complex interactions between plasma lipids and endothelium [4]. In these processes, the implication of oxidative stress, defined as an imbalance between free radical production and antioxidant defenses, is of great importance. Several studies reported that HFD causes oxidative burden in murine models [5-8]. However, Hogan et al. showed that a 12-week HFD induced obesity but did not significantly modify the plasma Oxygen Radical Absorbance Capacity (ORAC), a biomarker of antioxidant status, in male $\mathrm{C} 57 \mathrm{BL} / 6 \mathrm{~J}$ mice [9]. In the different previous published studies, the influence of HFD on vascular intimal thickening was mainly studied using histological approaches [10] and cardiac function 
was studied using either isolated hearts [11] or indirectly deduced from histological studies of aortic valves [12]. Finally, almost all studies concerning the development of plaques induced by HFD were conducted using males. It is well known that this type of diet influences less weight-gain in females than in males. The aim of the present study was to evaluate in adult female C57BL/6J strains, in which LDLR was present or deleted, the influence of a 17-week HFD on plasma antioxidant status and also on vascular wall thickening and cardiac function using high sensitivity echocardiography that proved to be an excellent tool for this purpose $[10,13,14]$.

\section{METHODS}

\subsection{Animal Models}

Female C57BL/6J wild type (WT) mice from Janvier (Saint Berthevin, France) and low-density lipoprotein receptor $\left(\mathrm{LDLR}^{-/}\right)$mice from The Jackson Laboratory (Bar Harbor, USA), 12 weeks old, were randomly divided into two different dietary regimens for 17 weeks. The normal diet (ND) group was fed standard pellets (Product \# A04 formulated by Safe; Augy, France). The high-fat diet (HFD) group was given a Western diet containing 40 $\mathrm{kcal} \%$ fat, $1.25 \%$ cholesterol and $0 \%$ cholic acid (Product \# D12108C formulated by Research Diets; New Brunswick, USA). Mice were maintained in a temperature-controlled $\left(22^{\circ} \mathrm{C}-25^{\circ} \mathrm{C}\right)$ room with a strict 12 -hour light/dark cycle and free access to food. This experimental study was approved by the local ethics committee and the investigators complied with authorization 21CAE057 from the French government, which agrees with the Directive 2010/63/EU of the European Parliament.

\subsection{Echocardiography}

Transthoracic micro-echocardiography was performed using a Vevo $770^{\circledR}$ (Visualsonics; Toronto, Canada) equipped with a 30-MHz high-frequency linear transducer. Mice were sedated with $2 \%$ isoflurane (Belamont, Cournon, France), then maintained at $1 \%$ and at a rectal temperature at $37^{\circ} \mathrm{C}-38^{\circ} \mathrm{C}$. The echocardiography examination was done using different views according to the guidelines of the American Society of Echocardiography [15] and the European Association of Echocardiography Recommendations [16]. Each measurement was obtained from five consecutive cardiac cycles and the average values were computed. Coefficient of variability of analyzed parameters was below $<10 \%$.

Cardiac imaging: B-Mode (2-D) images of the left ventricle (LV) were captured in the parasternal long and short axis views. LV internal diameters (LVID) were measured at end diastole (d) and systole (s) in M-mode. Heart rate (HR) was measured from the cardiac cycles recorded on the M-mode tracing, using 3 consecutive beats. LV mass $(\mathrm{LVm})$ was calculated from the LV wall thickness and chamber dimension obtained by M-mode and was normalized for body weight. LV volumes were calculated according to the M-mode formula of Teichholz et al. [17], and LV systolic function was analyzed by the measure of the ejection fraction (EF) of the LV. The cardiac output (CO) was calculated.

Vascular imaging: A right parasternal long axis view was used to assess the aortic arch morphology. External and internal diameters and walls thickness were measured in ascending aorta (Asc Ao), brachiocephalic artery $(\mathrm{BC})$, left common carotid artery $(\mathrm{CCa})$.

\subsection{Plasma Analysis}

One $\mathrm{mL}$ of blood was collected from mice anesthetized with sodium thiopental $(60 \mathrm{mg} / \mathrm{kg}$ intraperitoneal; Merial, Lyon, France) and treated with sodium heparin (500 UI/kg; Sanofi Aventis, Paris, France). Plasma was removed after centrifugation at $240 \mathrm{~g}$ for $10 \mathrm{~min}$ at $4^{\circ} \mathrm{C}$.

Lipid analysis: Total cholesterol (Total C), high-density lipoprotein cholesterol (HDL-C), low-density lipoprotein cholesterol (LDL-C) and triglyceride (TG) levels were measured using a colorimetric autoanalyzer (Dimension Vista ${ }^{\circledR}$ System). Atherogenic index (AI) was calculated by the formula: (Total C-HDL-C)/HDL-C.

Vitamin $\mathrm{C}$ and ascorbyl free radical analysis: For Vitamin C (Vit C) levels, samples (plasma and food: pelleted ND and HFD) were mixed with metaphosphoric acid $(5 \%)$ and then analyzed with High Performance Liquid Chromatography equipped with fluorescence detection (exc. $360 \mathrm{~nm}$; emission $440 \mathrm{~nm}$ ) [18]. Plasma Ascorbyl Free Radical (AFR) was detected by Electron Spin Resonance (ESR) spectroscopy [19]. Thirty-five $\mu 1$ of plasma were analyzed into a quartz capillary tube using a Bruker EMX X-band spectrometer (modulation frequency: $100 \mathrm{~Hz}$; amplitude modulation: $0.8 \mathrm{Ghz}$; conversion time: $40 \mathrm{~ms}$; scan time: $41 \mathrm{~s}$; 6 scans). The formation of AFR in plasma was determined from the height of the AFR signal intensity measured and expressed in arbitrary units (AU). The level of plasma oxidative stress was estimated by the AFR to Vit $\mathrm{C}$ ratio.

Total antioxidant capacity analyses: The total antioxidant activity of plasma was determined with an Oxygen Radical Absorbance Capacity (ORAC) miniaturized assay using 96-well microplates, a modified method of Cao et al. [20]. Fluorescence decay of Allophycocyanin (APC; Sigma) due to a peroxyl radical generator (AAPH, 2,2azobis(2-amidinopropane)-4-hydrochloride; Sigma) was measured over time, with excitation and emission filters of P620/8 and D665, respectively, using a Victor ${ }^{\circledR} 3 \mathrm{~V}$ fluorimeter 96-well microplate reader (Perkin Elmer). Trolox (6-Hydroxy-2,5,7,8-tetramethyl chroman-2-carboxylic acid; Sigma), a soluble vitamin E analog, was used as the antioxidant reference. Data was expressed as $\mu \mathrm{mol} / \mathrm{L}$ Trolox equivalents (TE). For each sample, the 
total antioxidant capacity measure was quadruplicated and averaged.

\subsection{Histology}

The entire heart with aortic arch, including ascending aorta (Asc Ao), brachiocephalic (BC) artery and left common carotid artery $(\mathrm{CCa})$, was collected and snapfrozen in isopentane then embedded in OCT compound. Histomorphometry was conducted on $6 \mu \mathrm{m}$ thick sections and realized using a microtome (CM3050, Leica microsystems, Germany). Samples were stained with Masson's Trichrome for light-microscope inspection (Eclipse 600, Nikon, Champigny-Sur-Marne, France) connected to a digital video camera (Tri CCD, Sony, France).

\subsection{Statistical Analysis}

Data are expressed as mean \pm SEM. Different groups were compared by analysis of variance (One-way ANOVA with Student-Newman-Keuls test). All statistical analyses were performed using SigmaPlot 11.0 (Systat software; San Jose, CA). Differences with a probability of less than $5 \%$ (values of $\mathrm{p}<0.05$ ) were considered statistically significant.

\section{RESULTS}

\subsection{Body Weight and Plasma Lipids}

In $\mathrm{LDLR}^{-/}$as in WT female mice, 17 weeks of HFD induced a $10 \%$ increase in body weight $(\mathrm{p}<0.01$, Table $\mathbf{1})$. Plasma levels of Total-C, LDL-C and TG did not differ between ND and HFD WT groups. However, an increase in HDL-C levels was observed in HFD WT as compared to ND WT mice $(+48 \%, \mathrm{p}=0.001)$. In ND groups, $\mathrm{LDLR}^{-/}$mice presented a 6-fold higher Total-C $(\mathrm{p}<$ $0.001), 1.5$-fold higher HDL-C $(\mathrm{p}<0.001)$ and 20 -fold higher LDL-C $(\mathrm{p}<0.001)$ plasma levels than WT mice.

The 17-weeks HFD in $\mathrm{LDLR}^{-/-}$mice led to a significant increase in Total-C $(p<0.001)$, HDL-C $(p=0.006)$,

Table 1. Effect of 17-week diet on body weight and plasma lipid levels in female wild type (WT) and $\mathrm{LDLR}^{-/}$mice.

\begin{tabular}{ccccc}
\hline & \multicolumn{2}{c}{ WT mice } & \multicolumn{2}{c}{ LDLR $^{-/}$mice } \\
\cline { 2 - 5 } & ND $(\mathrm{n}=8)$ & HFD $(\mathrm{n}=8)$ & $\mathrm{ND}(\mathrm{n}=8)$ & $\operatorname{HFD}(\mathrm{n}=7)$ \\
\hline Body Weight $(\mathrm{g})$ & $23.0 \pm 0.2$ & $25.4 \pm 0.6^{\mathrm{a}}$ & $22.8 \pm 0.4$ & $25.4 \pm 0.5^{\mathrm{a}}$ \\
Total C (mM) & $1.08 \pm 0.25$ & $1.71 \pm 0.35$ & $6.36 \pm 0.43^{\mathrm{b}}$ & $20.66 \pm 1.15^{\mathrm{a}, \mathrm{b}}$ \\
HDL-C (mM) & $0.58 \pm 0.09$ & $0.86 \pm 0.15^{\mathrm{a}}$ & $0.85 \pm 0.02^{\mathrm{b}}$ & $1.09 \pm 0.08^{\mathrm{a}, \mathrm{b}}$ \\
LDL-C (mM) & $0.15 \pm 0.08$ & $0.47 \pm 0.15$ & $3.07 \pm 0.16^{\mathrm{b}}$ & $10.79 \pm 0.49^{\mathrm{a}, \mathrm{b}}$ \\
TG (mM) & $0.32 \pm 0.09$ & $0.21 \pm 0.09$ & $0.78 \pm 0.23$ & $3.37 \pm 0.51^{\mathrm{a}, \mathrm{b}}$ \\
AI & $0.82 \pm 0.16$ & $0.98 \pm 0.15$ & $6.47 \pm 0.34^{\mathrm{b}}$ & $18.25 \pm 1.12^{\mathrm{a}, \mathrm{b}}$ \\
\hline
\end{tabular}

ND, normal diet; HFD, high fat diet, C, cholesterol; HDL-C, high-density lipoprotein cholesterol; LDL-C, low-density lipoprotein cholesterol; TG, triglyceride; AI, atherogenic index. Significant differences: HFD and ND were compared in each strain (WT or LDLR $\left.{ }^{-/}\right)\left({ }^{\mathrm{a}} \mathrm{p}<0.05\right)$; $\mathrm{LDLR}^{-/-}$and WT mice were compared in the same diet group $\left({ }^{\mathrm{b}} \mathrm{p}<0.05\right)$.
LDL-C $(\mathrm{p}<0.001)$ and TG $(\mathrm{p}<0.001)$ in comparison to $\mathrm{ND} \mathrm{LDLR}{ }^{-/}$mice. $\mathrm{LDLR}^{-/-}$mice have a higher $\mathrm{AI}$ in comparison with WT mice $(\mathrm{p}<0.001)$ and much higher after HFD $(p<0.001)$.

\subsection{Vascular and Aortic Arch Thickening}

Figure 1(A) shows some of the B-Mode echographic images of the Asc Ao, BC and CCa arteries in ND and HFD LDLR $^{-/}$mice used for the study. Morphometric studies were systematically conducted on the different images obtained with the different mice groups. For WT mice, no significant differences in arterial diameters and walls thickness were observed between ND and HFD groups (Figure 2). At the opposite, a significant increase in external diameters of Asc Ao $(+0.20 \mathrm{~mm}), \mathrm{BC}(+0.17$ $\mathrm{mm})$ and $\mathrm{CCa}(+0.08 \mathrm{~mm})$ arteries was noted in HFD LDLR $^{-/}$mice $(\mathrm{p}<0.001)$ when compared with ND $\mathrm{LDLR}^{-/}$or HFD WT mice. The lower wall of the Asc Ao $(p<0.001)$ and the upper wall of the BC $(p<0.001)$ and $\mathrm{CCa}(\mathrm{p}<0.01)$ arteries were significantly thicker in HFD $\mathrm{LDLR}^{-/}$than in ND $\mathrm{LDLR}^{-/-}$or HFD WT mice. The thickening of vessel wall in HFD LDLR $^{-/-}$mice was detected in the lower wall of the Asc Ao in 7 mice $(100 \%)$, in the upper wall of the $\mathrm{BC}$ artery in 6 mice $(86 \%)$ and in the upper wall of the $\mathrm{CCa}$ artery in 1 mouse (14\%). In every case, thickening was linked to a higher echogenicity.

Histology confirmed that $\mathrm{LDLR}^{-/}$mice fed a HFD developed large atherosclerotic lesions in the aortic arch (Figure 1(B)). No lesion was found in $\mathrm{LDLR}^{-/}$mice fed a standard chow diet. WT mice fed a ND or HFD never developed any lesions (data not shown). Histological analyses of aortic valves sections (Figure 3) did not show any thickening of intima-media in WT mice fed a HFD. At the opposite in $\mathrm{LDLR}^{-/}$mice, HFD significantly increased aortic valve atherosclerotic lesions areas. It must be noted that ND-fed $\mathrm{LDLR}^{-/-}$mice present larger and thicker aortic valves than WT mice.

\subsection{Plasma Oxidative Stress Biomarkers}

Plasma ORAC values were similar in ND-fed WT and $\mathrm{LDLR}^{-/-}$mice. HFD induced a slight but significant decrease in ORAC in WT mice $(p=0.011$, Table 2$)$ while no significant differences were noted in $\mathrm{LDLR}^{-/-}$mice.

Circulating Vit $\mathrm{C}$ was not significantly different between ND-fed WT and $\mathrm{LDLR}^{-1-}$ mice. We clearly observed that HFD induced a significant increase of Vit C levels in both groups, but much higher in $\mathrm{LDLR}^{-/-}(1.7$ fold increase, $p<0.001$ ). We thus checked that Vit $C$ was neither detected in ND nor in HFD pellets (data not shown).

We also focused our interest on Ascorbyl Free Radicals (AFR) levels in plasma. AFR was significantly increased by HFD in WT mice $(p<0.05)$ but the increase 
Normal Diet
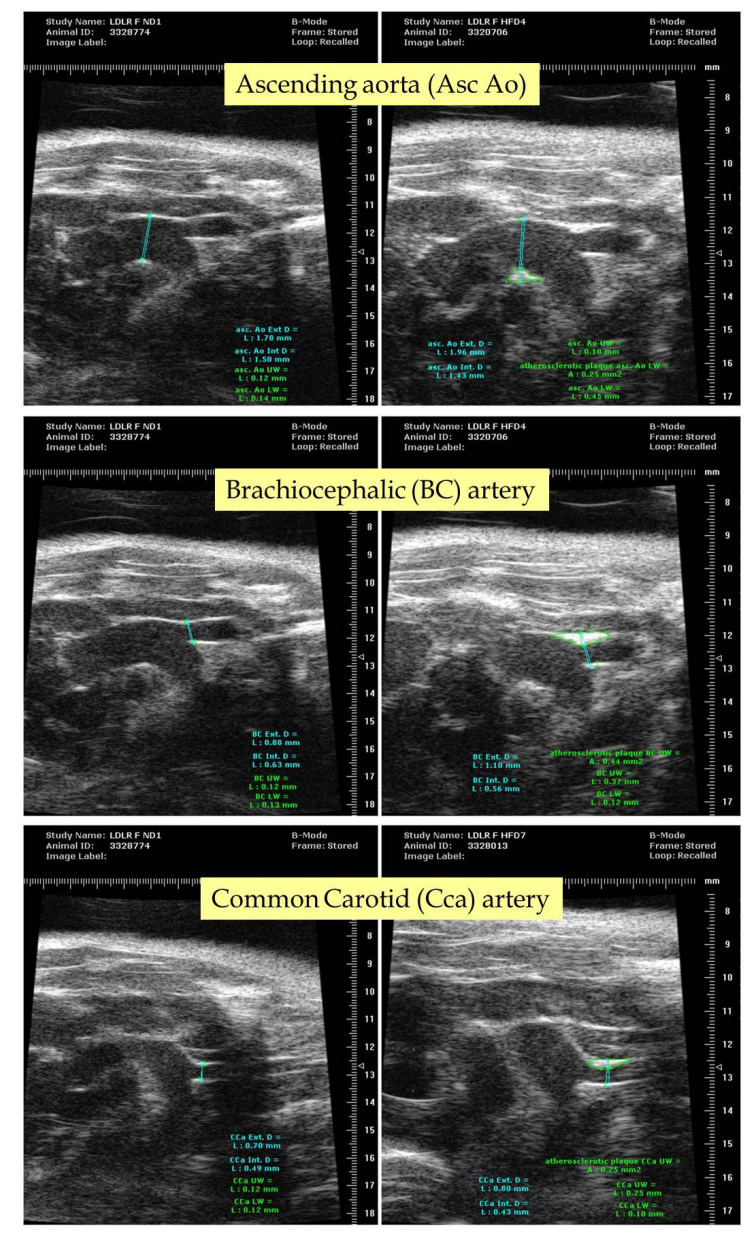

(B)
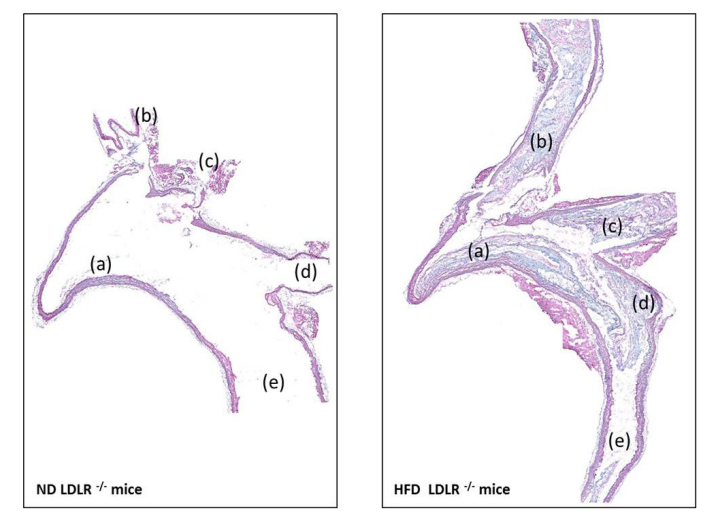

Figure 1. (A) Ultrasound images of the aortic arch of female $\mathrm{LDLR}^{-/-}$mice fed 17-week normal diet or high fat diet. Arch is observed in its long-axis from the aortic valves to the mid arch. For morphometry, external and internal diameters, upper and lower wall thickness of artery vessels were measured using B-Mode images of the right parasternal view of the aortic arch; (B) Low magnification histological views of longitudinal sections of aortic arch from 2 sacrificed $\mathrm{LDLR}^{--}$females showing the effect of 17-week diet (left: fed a normal diet (ND) and right: fed HFD). (a) Ascending aorta; (b) Brachocephalic artery; (c) Left common carotid artery; (d) Left subclavian artery; (e) Descending thoracic aorta. was not statistically significant in $\mathrm{LDLR}^{-/-}$mice. The estimated AFR/Vit $\mathrm{C}$ ratio, an endogenous oxidative stress index, is decreased in both mice stains by HFD but with statistical significance for $\operatorname{LDLR}^{-1-}$ mice only $(\mathrm{p}=$ 0.011 ), probably due to higher increase observed in Vit $\mathrm{C}$ level.

\subsection{Echographic Evaluation of Left Ventricular Function}

Results are presented in Table 3. First, HR was similar in WT and $\mathrm{LDLR}^{-/-}$mice when fed a ND. HFD did not influence this value in WT mice, while in $\mathrm{LDLR}^{-1-}$ mice, HR was much higher than in all the other groups: compared with ND group $(\mathrm{p}=0.019)$ or with HFD WT mice $(\mathrm{p}=0.002)$.

LVIDd and LVIDs measured in parasternal long and short axis views did not differ between the 4 groups. Correlatively, there was no significant difference between the estimated LV mass (LVm) of the different groups. Because body weight (BW) was increased by $\mathrm{HFD}$, the LVm to BW ratio was decreased in WT mice ( $\mathrm{p}$ $=0.007)$. At the opposite, this ratio did not varied in $\mathrm{LDLR}^{-/-}$mice when fed with HFD.

No difference among the 4 groups was noted for $\mathrm{CO}$. However, EF was significantly slightly increased by the HFD both in WT $(p=0.043)$ and $\operatorname{LDLR}^{-/}(p=0.012)$ mice.

\section{DISCUSSION}

\subsection{Effect of HFD on Circulating Lipids and Body Weight}

Hyperlipidemia is one of the major risk factors for cardiovascular diseases and Low-density lipoprotein cholesterol (LDL-C) is considered as a key factor for the induction of intimal plaques. Excess of LDL-C accumulates in large conductance arteries intima then, after undergoing oxidation, promotes the formation of atherosclerotic plaque. Conversely, high-density lipoprotein cholesterol (HDL-C) is recognized as beneficial. Elevated levels of HDL-C, exert a protective role against the development of atherosclerotic plaques due to their anti-inflammatory [21] and antioxidant [22] properties, their increase of cholesterol metabolism in liver, and their inhibitory action on LDL oxidation and secretion of adhesion molecules by endothelial cells [22,23]. The HDL to LDL ratio, reflected by $\mathrm{AI}$, is considered to be a good prognostic indicator for atherosclerosis extent [24]. In our study, only $\mathrm{LDLR}^{-/-}$mice fed a HFD presented an increased AI. In WT mice, the circulating levels of HDL, almost two times higher as compared to LDL-C levels, could probably lead to inhibition of LDL oxidation and induce a protection against the development of atherosclerotic plaque. At the opposite, in $\mathrm{LDLR}^{-/}$mice, 

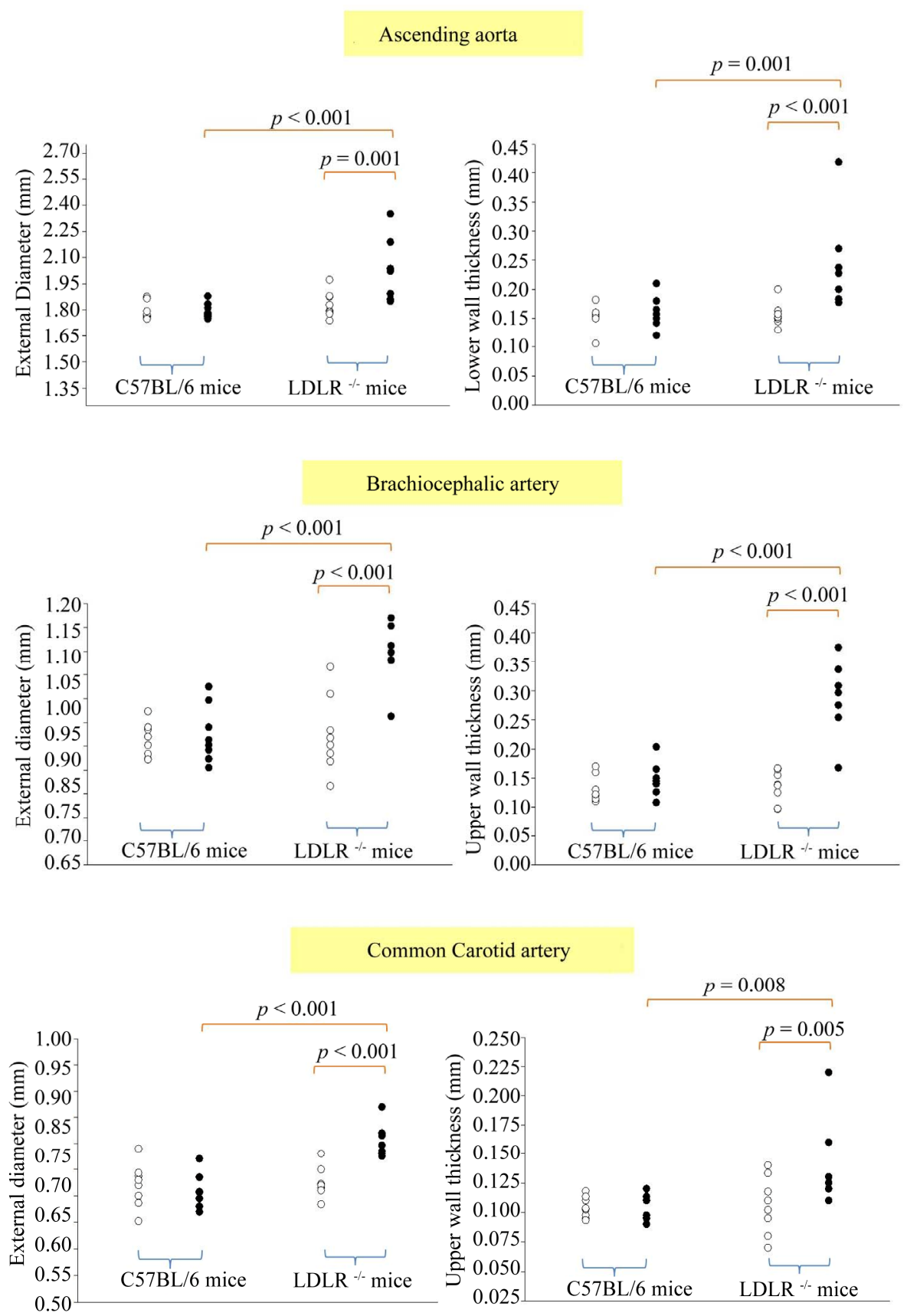

Figure 2. Morphometric study of the effect of diet on external diameter and wall thickness of aortic arch, measured using echocardiography, in female wild type (WT) and $\mathrm{LDLR}^{-1}$ mice. WT and LDLR $^{-1}$ mice were fed a normal diet (ND) or high-fat diet (HFD) for 17 weeks.

although plasma HDL-C levels were more important in HFD than in ND groups, their levels 10 times lower compared to LDL-C could explain that HDL-C cannot inhibit the LDL-C oxidative modification.

In our study, 17-week cholesterol-enriched diet induced a similar weight gain in WT and in $\mathrm{LDLR}^{-/-}$mice aged 12 weeks old at the beginning of the diet. This weight increase is moderate $(10 \%)$ and we observed in another study that it was similar in males of the same age
(Alban Dupoux, unpublished results). If older mice (10 months old) are fed a HFD during the same period, a more pronounced weight increase is observed in males as in females (approximately 18\%, unpublished data Loranne Maugé). Thus, although plasma lipids were profoundly modified (20 fold increase in Total Cholesterol, 10 fold for TG and 72 fold for LDL) in LDLR $^{-/}$fat fed females compared to basal levels in chow diet fed WT mice, the weight gain was rather limited. 


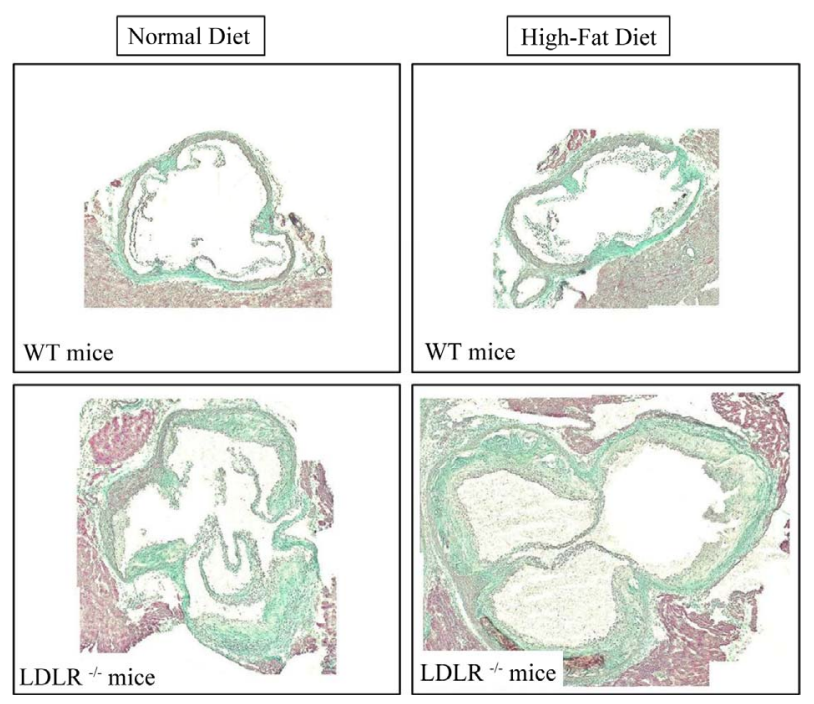

Figure 3. Representative pictures of aortic valves sections stained with Masson's Trichrome at the same magnification (lens $\times 10$ ): Effect of 17 weeks HFD on aortic valves morphology in female wild type (WT) and $\mathrm{LDLR}^{-/}$mice.

Table 2. Effect of 17-week diet on plasma oxidative stress in female wild type (WT) and $\mathrm{LDLR}^{-/-}$mice.

\begin{tabular}{lcccc}
\hline & \multicolumn{2}{c}{ WT mice } & \multicolumn{2}{c}{ LDLR $^{-/-}$mice } \\
\cline { 2 - 5 } & $\begin{array}{c}\text { ND } \\
(\mathrm{n}=8)\end{array}$ & $\begin{array}{c}\text { HFD } \\
(\mathrm{n}=8)\end{array}$ & $\begin{array}{c}\text { ND } \\
(\mathrm{n}=8)\end{array}$ & $\begin{array}{c}\text { HFD } \\
(\mathrm{n}=7)\end{array}$ \\
\hline ORAC $(\mu \mathrm{mol} / \mathrm{L} \mathrm{TE})$ & $2037 \pm 79$ & $1611 \pm 105^{\mathrm{a}}$ & $2212 \pm 94$ & $2533 \pm 154^{\mathrm{a}}$ \\
$\operatorname{VitC}(\mu \mathrm{mol} / \mathrm{L})$ & $53 \pm 3$ & $79 \pm 3^{\mathrm{a}}$ & $61 \pm 6$ & $104 \pm 8^{\mathrm{a}, \mathrm{b}}$ \\
$\mathrm{AFR}(\mathrm{AU})$ & $1.6 \pm 0.0$ & $2.2 \pm 0.1^{\mathrm{a}}$ & $2.1 \pm 0.2$ & $2.5 \pm 0.3$ \\
$\begin{array}{l}\mathrm{AFR}(\mathrm{AU}) / \mathrm{VitC} \\
(\mu \mathrm{mol} / \mathrm{L})\left(\times 10^{-2}\right)\end{array}$ & $3.18 \pm 0.19$ & $2.85 \pm 0.14$ & $3.65 \pm 0.25$ & $2.47 \pm 0.21^{\mathrm{a}}$ \\
\hline
\end{tabular}

ND, normal diet; HFD, high fat diet, Antioxidant activity was determined by ORAC (Oxygen Radical Absorbance Capacity) and was expressed in $\mu \mathrm{mol} / \mathrm{L}$ Trolox equivalent (TE). Vit C, vitamin C; AFR, ascorbyl free radical expressed in arbitrary units (AU). Significant differences: HFD and ND were compared in each strain (WT or $\left.\mathrm{LDLR}^{-1-}\right)\left({ }^{\mathrm{a}} \mathrm{p}<0.05\right) ; \mathrm{LDLR}^{-/-}$and WT mice were compared in the same diet group $\left({ }^{\mathrm{b}} \mathrm{p}<0.05\right)$.

Table 3. Effect of 17-week diet on cardiac function evaluated by echocardiography in female wild (WT) and $\mathrm{LDLR}^{-/-}$mice.

\begin{tabular}{ccccc}
\hline & \multicolumn{2}{c}{ WT mice } & \multicolumn{2}{c}{ LDLR $^{-/-}$mice } \\
\cline { 2 - 5 } & $\mathrm{ND}(\mathrm{n}=8)$ & HFD $(\mathrm{n}=8)$ & $\mathrm{ND}(\mathrm{n}=8)$ & HFD $(\mathrm{n}=7)$ \\
\hline HR, beats/min & $449 \pm 11$ & $447 \pm 12$ & $457 \pm 11$ & $515 \pm 16^{\mathrm{a}, \mathrm{b}}$ \\
LVIDd, mm & $3.92 \pm 0.04$ & $3.79 \pm 0.05^{\mathrm{a}}$ & $3.85 \pm 0.05$ & $3.77 \pm 0.77$ \\
LVIDs, mm & $2.82 \pm 0.06$ & $2.60 \pm 0.07$ & $2.82 \pm 0.05$ & $2.63 \pm 0.07$ \\
LVm, mg & $104 \pm 4$ & $96 \pm 2$ & $98 \pm 3$ & $109 \pm 5$ \\
LVm/BW & $4.5 \pm 0.2$ & $3.8 \pm 0.1^{\mathrm{a}, \mathrm{b}}$ & $4.3 \pm 0.2$ & $4.3 \pm 0.2$ \\
$\mathrm{CO}, \mathrm{mL} / \mathrm{min}$ & $17 \pm 1$ & $16 \pm 1$ & $15 \pm 1$ & $18 \pm 1$ \\
$\mathrm{EF}, \%$ & $55 \pm 1$ & $60 \pm 2^{\mathrm{a}}$ & $53 \pm 1$ & $58 \pm 1^{\mathrm{a}}$ \\
\hline
\end{tabular}

ND, normal diet; HFD, high fat diet; HR, heart rate; LVIDd, Left Ventricular Internal diameter at end diastole; LVIDs, Left Ventricular Internal diameter at end systole; EF, ejection fraction; LVm, Left Ventricular mass; CO, Cardiac Output. Significant differences: HFD and ND were compared in each strain (WT or $\left.\mathrm{LDLR}^{-1}\right)\left({ }^{\mathrm{a}} \mathrm{p}<0.05\right)$; $\mathrm{LDLR}^{-/}$and WT mice were compared in the same diet group $(\mathrm{b}<0.05)$.

\subsection{Effect of HFD on Plasma Oxidant/Antioxidant Status}

Vit $\mathrm{C}$ acts as a major plasma antioxidant to regulate redox status [25]. Our results clearly demonstrated that 17 weeks of HFD induced an increase in plasma Vit $\mathrm{C}$ levels. In $\mathrm{LDLR}^{-/-}$mice fed a HFD, the enhancement in Vit $\mathrm{C}$ is more pronounced. This could be explained because at the opposite of humans, mice are able to synthesize Vit C from glucose using gulonolactone oxidase. Thus, because no amount of Vit $\mathrm{C}$ was found in the pellets of normal and high-fat diet, the enhanced levels of Vit $\mathrm{C}$ could be due to an increase in endogenous synthesis by the liver. However a decrease in its renal excretion could also be considered. Nakata and Maeda [26] developed the hypothesis that, in human, deficiency in Vit $\mathrm{C}$ could be responsible for early development of atherosclerotic plaques, but established in gulonolactone and apolipoprotein E-deficient mice that Vit $\mathrm{C}$ is not involved in the early steps of and apolipoprotein E-deficient but played a role in plaque stabilization [26]. In our study, we observed an increase in plaque development, after 17 weeks HFD, in a context of increased Vit $\mathrm{C}$ level, suggesting that Vit $\mathrm{C}$ did not succeeded to limit plaque development.

We also noted that although Vit $\mathrm{C}$ levels are increased, AFR to Vit $\mathrm{C}$ ratio, considered as an indicator of plasma endogenous oxidative status [27], decreased in both mice strains showing that up-regulation of Vit $\mathrm{C}$ levels is not sufficient to protect efficiently against atherosclerotic plaque development in the HFD-fed mice. In fact, the global antioxidative activity, estimated by the plasma ORAC value, was significantly decreased by HFD in WT mice. At the opposite, in $\mathrm{LDLR}^{-/}$mice, in which plasma lipid levels were considerably disturbed, ORAC value was rescued. This could be due to the high levels of Vit $\mathrm{C}$ that were described to induce antioxidant stress proteins and protect LDL from oxidation $[28,29]$. This could also be due to the HFD-induced hypertriglyceridemia in $\mathrm{LDLR}^{-1-}$ mice that could chronically influence oxidative stress via activation of mitochondrial respiratory chain and production of free radicals [30-32]. Consequently, HFD could constitute in $\mathrm{LDLR}^{--}$mice an endogenous preconditioning by oxidative radicals that stimulates antioxidative defences as already suggested [11].

\subsection{Effect of HFD on Aortic Arch Morphology}

Our high quality images, obtained using echography, allowed to demonstrate on living animals a significant increase in external diameter and upper wall thickness of ascending aorta, brachiocephalic artery and common carotid artery of LDLR ${ }^{-/}$mice fed a HFD. This effect of HFD was not evidenced in WT in which the diet did not induced profound plasma lipid levels perturbations. In accordance with these results, histology showed major 
lesions in aortic arch in HFD-fed $\mathrm{LDLR}^{-/-}$mice but not under standard diet as already shown by Ishibashi et al. [3] in sacrificed animals. This is also in accordance with the fact that these regions represent predilection sites for atherosclerotic development [33]. Furthermore, our histological study in aortic arch demonstrates that the increased echogenicity of wall intimal thickening probably corresponded to collagenous lesions.

Interestingly, a thickening of vascular walls was observed but the internal diameter of vessels was preserved in HFD-fed $\mathrm{LDLR}^{-1-}$ mice while external diameter was increased. Several studies have reported similar results regarding the enlargement of vessel in plaque sites using magnetic resonance or intravascular ultrasound imaging $[34,35]$. This enlargement is described as a compensatory response for intimal hyperplasia formation that may lead to vascular expansive remodeling [36]. It is thus not surprising that these lesions are associated to intact cardiac function in HFD LDLR ${ }^{-/}$mice, since the thickening of the wall does not seem to have a significant impact on the luminal diameter of the vessels and does not significantly obstruct the flow.

\subsection{Effect of HFD on Aortic Valves and Cardiac Function}

After 17 weeks on a Western-type diet, LDLR $^{-/}$mice showed an increase in aortic valves atherosclerotic lesions. Intimal lesions present extensive areas of collagen and cholesterol crystals accumulation typically observed in many other studies $[12,37,38]$ and demonstrating well established plaques. Drolet et al., already reported that 16 weeks of normal or high-fat/high-carbohydrate diet induces significant aortic valve abnormalities both ex vivo (leaflet thickening) and in vivo by echocardiography-doppler (smaller valve area and higher transvalvular velocities), but did not gave indication on consequences of the presence of these lesions on cardiac function of these mice [39].

In human as in mice, systolic dysfunction is considered as significant from EF $<50 \%$. Our study, using echocardiography, shows that chronic HFD significantly induced a very moderate increase in the EF. However, EF of the mice always ranged between $55 \%$ and $60 \%$. We also observed similar values after a shorter duration of diet ( 9 weeks, data not shown). Thus HFD did not significantly modify cardiac function of these mice.

Despite of this apparent lack of significant effect on mice fed a HFD, in view of their higher body weight, their lack of noticeable cardiac hypertrophy, and in view that we did not checked their diastolic function in this study, they could perhaps develop heart failure with preserved systolic function as it is observed with some drugs cardiotoxicity [40].

\section{CONCLUSION}

This study demonstrates that serious disturbances in lipid plasma levels are associated to variations of circulating antioxidant status due, at least in part to increased levels of plasma Vit $\mathrm{C}$ in mice fed a HFD. Atherosclerotic lesions observed in aortic arch and in aortic valve do not impair cardiac function in mice fed a HFD.

\section{ACKNOWLEDGEMENTS}

We thank Dr A. BOUCHOT and A. BATAILLE (CellimaP, IFR100 Santé-STIC, University of Burgundy, France) for sectioning and staining of tissues, Dr M. ZELLER (Laboratory of Cardio-Metabolic Pathophysiology and Pharmacology, University of Burgundy, Inserm UMR866, France) and Dr C. TOUZERY (Department of Cardiology, University Hospital, Dijon, France) for statistical assistance. Vevo $770^{\circledR}$ echocardiograph was funded by European Community Funds (FEDER), Burgundy Regional Council and French Ministry for Research (MESR). Victor ${ }^{\circledR}$ reader (Perkin Elmer) was funded by French Ministry for Research (MESR).

\section{REFERENCES}

[1] Damjanovic, M. and Barton, M. (2008) Fat intake and cardiovascular response. Current Hypertension Reports, 10, 25-31. doi:10.1007/s11906-008-0007-0

[2] Ishibashi, S., Brown, M.S., Goldstein, J.L., et al. (1993) Hypercholesterolemia in low density lipoprotein receptor knockout mice and its reversal by adenovirus-mediated gene delivery. Journal of Clinical Investigation, 92, 883893. doi:10.1172/JCI116663

[3] Ishibashi, S., Goldstein, J.L., Brown, M.S., et al. (1994) Massive xanthomatosis and atherosclerosis in cholesterol-fed low density lipoprotein receptor-negative mice. Journal of Clinical Investigation, 93, 1885-1893. doi:10.1172/JCI117179

[4] Hansson, G.K. (2005) Inflammation, atherosclerosis, and coronary artery disease. The New England Journal of Medicine, 352, 1685-1695. doi:10.1056/NEJMra043430

[5] Zhu, X., Zhang, W., Pang, X., et al. (2011) Hypolipidemic effect of n-butanol extract from Asparagus officinalis L. in mice fed a high-fat diet. Phytotherapy Research, 25, 1119-1124. doi:10.1002/ptr.3380

[6] Azuma, T., Tomofuji, T., Endo, Y., et al. (2011) Effects of exercise training on gingival oxidative stress in obese rats. Archives of Oral Biology, 56, 768-774.

doi:10.1016/j.archoralbio.2011.01.008

[7] Ruggiero, C., Ehrenshaft, M., Cleland, E., et al. (2011) High-fat diet induces an initial adaptation of mitochondrial bioenergetics in the kidney despite evident oxidative stress and mitochondrial ROS production. American Journal of Physiology-Endocrinology and Metabolism, 300, E1047-E1058. doi:10.1152/ajpendo.00666.2010

[8] Salvati, S., Attorri, L., Di Benedetto, R., et al. (2011) Micronutrient-enriched rapeseed oils improve the brain oxidant/antioxidant system in rats fed a high-fat diet. 
Journal of Agricultural and Food Chemistry, 59, 44834488. doi:10.1021/jf1042807

[9] Hogan, S., Canning, C, Sun, S., et al. (2011) Dietary supplementation of grape skin extract improves glycemia and inflammation in diet-induced obese mice fed a western high fat diet. Journal of Agricultural and Food Chemistry, 59, 3035-3041. doi:10.1021/jf1042773

[10] Gan, L.M., Gronros, J., Hagg, U., et al. (2007) Non-invasive real-time imaging of atherosclerosis in mice using ultrasound biomicroscopy. Atherosclerosis, 190, 313-320. doi:10.1016/j.atherosclerosis.2006.03.035

[11] Lauzier, B., Delemasure, S., Pesant, M., Collin, B., Duvillard, L., Vergely, C., Connat, J.-L. and Rochette, L. (2009) A cholesterol-rich diet improves resistance to ischemic insult in mouse hearts but suppresses the beneficial effect of post-conditioning. Journal of Heart and Lung Transplantation, 28, 821-826. doi:10.1016/j.healun.2009.04.030

[12] Boisvert, W.A., Spangenberg, J. and Curtiss, L.K. (1997) Role of leukocyte-specific LDL receptors on plasma lipoprotein cholesterol and atherosclerosis in mice. Arteriosclerosis, Thrombosis, and Vascular Biology, 17, 340347. doi:10.1161/01.ATV.17.2.340

[13] Rottman, J.N., Ni, G. and Brown, M. (2007) Echocardiographic evaluation of ventricular function in mice. Echocardiography, 24, 83-89. doi:10.1111/j.1540-8175.2006.00356.x

[14] Gronros, J., Wikstrom, J., Brandt-Eliasson, U., et al. (2008) Effects of rosuvastatin on cardiovascular morphology and function in an ApoE-knockout mouse model of atherosclerosis. American Journal of PhysiologyHeart and Circulatory Physiology, 295, H2046-H2053. doi:10.1152/ajpheart.00133.2008

[15] Schiller, N.B., Shah, P.M., Crawford, M., et al. () Recommendations for quantitation of the left ventricle by two-dimensional echocardiography. American Society of Echocardiography Committee on Standards, Subcommittee on Quantitation of Two-Dimensional Echocardiograms. Journal of the American Society of Echocardiography, 2, 358-367.

[16] Evangelista, A., Flachskampf, F.A., Erbel, R., et al. (2010) Echocardiography in aortic diseases: EAE recommendations for clinical practice. European Journal of Echocardiography, 11, 645-658. doi:10.1093/ejechocard/jeq056

[17] Teichholz, L.E., Kreulen, T., Herman, M.V., et al. (1976) Problems in echocardiographic volume determinations: Echocardiographic-angiographic correlations in the presence of absence of asynergy. American Journal of Cardiology, 37, 7-11. doi:10.1016/0002-9149(76)90491-4

[18] Tessier, F., Birlouez-Aragon, I., Tjani, C., et al. (1996) Validation of a micromethod for determining oxidized and reduced vitamin $\mathrm{C}$ in plasma by HPLC-fluorescence. International Journal for Vitamin and Nutrition Research, 66, 166-170.

[19] Richard, C., Lauzier, B., Delemasure, S., et al. (2008) Effects of angiotensin-1 converting enzyme inhibition on oxidative stress and bradykinin receptor expression during doxorubicin-induced cardiomyopathy in rats. Journal of Cardiovascular Pharmacology, 52, 278-285.
doi:10.1097/FJC.0b013e3181865f28

[20] Cao, G., Alessio, H.M. and Cutler, R.G. (1993) Oxygenradical absorbance capacity assay for antioxidants. Free Radical Biology and Medicine, 14, 303-311. doi:10.1016/0891-5849(93)90027-R

[21] Barter, P.J., Nicholls, S., Rye, K.A., et al. (2004) Antiinflammatory properties of HDL. Circulation Research, 95, 764-772. doi:10.1161/01.RES.0000146094.59640.13

[22] Podrez, E.A. (2010) Anti-oxidant properties of high-density lipoprotein and atherosclerosis. Clinical and Experimental Pharmacology and Physiology, 37, 719-725. doi:10.1111/j.1440-1681.2010.05380.x

[23] Bursill, C.A., Castro, M.L., Beattie, D.T., et al. (2010) High-density lipoproteins suppress chemokines and chemokine receptors in vitro and in vivo. Arteriosclerosis, Thrombosis, and Vascular Biology, 30, 1773-1778. doi:10.1161/ATVBAHA.110.211342

[24] Millan, J., Pinto, X., Munoz, A., et al. (2009) Lipoprotein ratios: Physiological significance and clinical usefulness in cardiovascular prevention. Journal of Vascular Health and Risk Management, 5, 757-765.

[25] Farbstein, D., Kozak-Blickstein, A. and Levy, A.P. (2010) Antioxidant vitamins and their use in preventing cardiovascular disease. Molecules, 15, 8098-8110. doi:10.3390/molecules 15118098

[26] Nakata, Y. and Maeda, N. (2002) Vulnerable atherosclerotic plaque morphology in apolipoprotein E-deficient mice unable to make ascorbic Acid. Circulation, 105, 1485-1490. doi:10.1161/01.CIR.0000012142.69612.25

[27] Buettner, G.R. and Jurkiewicz, B.A. (1993) Ascorbate free radical as a marker of oxidative stress: An EPR study. Free Radical Biology and Medicine, 14, 49-55. doi:10.1016/0891-5849(93)90508-R

[28] Retsky, K.L., Freeman, M.W. and Frei, B. (1993) Ascorbic acid oxidation product(s) protect human low density lipoprotein against atherogenic modification. Anti-rather than prooxidant activity of vitamin $\mathrm{C}$ in the presence of transition metal ions. Journal of Biological Chemistry, 268, 1304-1309.

[29] Siow, R.C., Sato, H., Leake, D.S., et al. (1999) Induction of antioxidant stress proteins in vascular endothelial and smooth muscle cells: Protective action of vitamin C against atherogenic lipoproteins. Free Radical Research, 31, 309-318. doi:10.1080/10715769900300871

[30] Gao, X., Zhao, X.L., Zhu, Y.H. et al. (2011) Tetramethylpyrazine protects palmitate-induced oxidative damage and mitochondrial dysfunction in $\mathrm{C} 2 \mathrm{C} 12$ myotubes. Life Sciences, 88, 803-809. doi:10.1016/j.lfs.2011.02.025

[31] Ruggiero, C., Ehrenshaft, M., Cleland, E. and Stadler, K. (2011) High-fat diet induces an initial adaptation of mitochondrial bioenergetics in the kidney despite evident oxidative stress and mitochondrial ROS production. American Journal of Physiology-Endocrinology and Metabolism, 300, E1047-E1058. doi:10.1152/ajpendo.00666.2010

[32] Vial, G., Dubouchaud, H., Couturier, K., Cottet-Rousselle, C., Taleux, N., Athias, A., Galinier, A., Castteilla, L. and Leverne, X.M. (2011) Effects of a high-fat diet on energy 
metabolism and ROS production in rat liver. Journal of Hepatology, 54, 348-356. doi:10.1016/j.jhep.2010.06.044

[33] VanderLaan, P.A., Reardon, C.A., Getz, G.S., et al. (2004) Site specificity of atherosclerosis: Site-selective responses to atherosclerotic modulators. Arteriosclerosis, Thrombosis, and Vascular Biology, 24, 12-22. doi:10.1161/01.ATV.0000105054.43931.f0

[34] Li, H.Y., Tanaka, K., Oeser, B., et al. (2006) Compensatory enlargement in transplant coronary artery disease: An intravascular ultrasound study. Chinese Medical Journal (English Edition), 119, 564-569.

[35] Bertini, P.J., Parga, J.R., Chagas, A.C., et al. (2005) Compensatory enlargement of human coronary arteries identified by magnetic resonance imaging. Brazilian Journal of Medical and Biological Research, 38, 661-667. doi:10.1590/S0100-879X2005000500002

[36] Schoenhagen, P., Ziada, K.M., Vince, D.G., et al. (2001) Arterial remodeling and coronary artery disease: The concept of "dilated" versus "obstructive" coronary atherosclerosis. Journal of the American College of Cardiology, 38, 297-306. doi:10.1016/S0735-1097(01)01374-2
[37] Bjorkbacka, H., Kunjathoor, V.V., Moore, K.J., et al. (2004) Reduced atherosclerosis in MyD88-null mice links elevated serum cholesterol levels to activation of innate immunity signaling pathways. Nature Medicine, 10, 416-421. doi:10.1038/nm1008

[38] Choi, S.E., Jang, H.J., Kang, Y., et al. (2010) Atherosclerosis induced by a high-fat diet is alleviated by lithium chloride via reduction of VCAM expression in ApoE-deficient mice. Vascular Pharmacology, 53, 264-272. doi:10.1016/j.vph.2010.09.004

[39] Drolet, M.C., Roussel, E., Deshaies, Y., et al. (2006) A high fat/high carbohydrate diet induces aortic valve disease in $\mathrm{C} 57 \mathrm{BL} / 6 \mathrm{~J}$ mice. Journal of the American College of Cardiology, 47, 850-855. doi:10.1016/j.jacc.2005.09.049

[40] Nagy, A.C., Cserep, Z., Tolnay, E., et al. (2008) Early diagnosis of chemotherapy-induced cardiomyopathy: A prospective tissue Doppler imaging study. Pathology \& Oncology Research, 14, 69-77. doi:10.1007/s12253-008-9013-4 